\title{
LERAY-SCHAUDER RESULTS FOR MULTIVALUED NONLINEAR CONTRACTIONS DEFINED ON CLOSED SUBSETS OF A FRÉCHET SPACE
}

\author{
RAVI P. AGARWAL AND DONAL O’REGAN
}

Received 9 December 2005; Revised 21 February 2006; Accepted 25 April 2006

New Leray-Schauder results are presented for multivalued contractions defined on subsets of a Fréchet space $E$. The proof relies on fixed point results in Banach spaces and on viewing $E$ as the projective limit of a sequence of Banach spaces.

Copyright (c) 2006 Hindawi Publishing Corporation. All rights reserved.

\section{Introduction}

In this paper, we present new fixed point results for nonlinear contractions (both single and multivalued) defined on subsets $X$ (which may have empty interior) of a Fréchet space $E$. Some results for single-valued maps were presented in $[2,3]$ and the approach in these papers was based on constructing a specific map $F_{n}$ (for each $n \in \mathbb{N}=\{1,2, \ldots\}$ ) whose fixed points converge to a fixed point of the original operator $F$. In the approach in this paper, the maps $\left\{F_{n}\right\}_{n \in \mathbb{N}}$ only need to satisfy a closure property and are specified in a completely different way. The advantage of this approach is that multivalued maps can also be discussed. Our theory is based on results in Banach spaces and on viewing a Fréchet space $E$ as a projective limit of a sequence of Banach spaces $\left\{E_{n}\right\}_{n \in \mathbb{N}}$.

For the remainder of this section, we present some definitions and some known facts. Let $(X, d)$ be a metric space and $S$ a nonempty subset of $X$. For $x \in X$, let $d(x, S)=$ $\inf _{y \in S} d(x, y)$. Also $\operatorname{diam} S=\sup \{d(x, y): x, y \in S\}$. We let $B(x, r)$ denote the open ball in $X$ centered at $x$ of radius $r$ and by $B(S, r)$ we denote $\bigcup_{x \in S} B(x, r)$. For two nonempty subsets $S_{1}$ and $S_{2}$ of $X$, we define the generalized Hausdorff distance $H$ to be

$$
H\left(S_{1}, S_{2}\right)=\inf \left\{\epsilon>0: S_{1} \subseteq B\left(S_{2}, \epsilon\right), S_{2} \subseteq B\left(S_{1}, \epsilon\right)\right\}
$$

Now suppose $G: S \rightarrow 2^{X}$; here $2^{X}$ denotes the family of nonempty subsets of $X$. Then $G$ is said to be hemicompact if each sequence $\left\{x_{n}\right\}_{n \in \mathbb{N}}$ in $S$ has a convergent subsequence whenever $d\left(x_{n}, G\left(x_{n}\right)\right) \rightarrow 0$ as $n \rightarrow \infty$.

We now recall a result from the literature. 
Theorem 1.1. Let $(X, d)$ be a complete metric space, $\mathbb{C} \subseteq X$ closed, and $F: \mathbb{C} \rightarrow X$ with $F(\mathbb{C})$ bounded (i.e., there exists $M>0$ with $d(z, w) \leq M$ for $z, w \in F(\mathbb{C})$ ). Suppose the following condition is satisfied:

$$
\begin{aligned}
& \text { there exists a continuous nondecreasing function } \\
& \phi:[0, \infty) \longrightarrow[0, \infty) \text { satisfying } \phi(z)<z \text { for } z>0 \\
& \text { such that } d(F x, F y) \leq \phi(d(x, y)) \text { for } x, y \in \mathbb{C} .
\end{aligned}
$$

Then $F$ is hemicompact.

Now let $I$ be a directed set with order $\leq$ and let $\left\{E_{\alpha}\right\}_{\alpha \in I}$ be a family of locally convex spaces. For each $\alpha \in I, \beta \in I$ for which $\alpha \leq \beta$, let $\pi_{\alpha, \beta}: E_{\beta} \rightarrow E_{\alpha}$ be a continuous map. Then the set

$$
\left\{x=\left(x_{\alpha}\right) \in \prod_{\alpha \in I} E_{\alpha}: x_{\alpha}=\pi_{\alpha, \beta}\left(x_{\beta}\right) \forall \alpha, \beta \in I, \alpha \leq \beta\right\}
$$

is a closed subset of $\prod_{\alpha \in I} E_{\alpha}$ and is called the projective limit of $\left\{E_{\alpha}\right\}_{\alpha \in I}$ and is denoted by $\lim _{\leftarrow} E_{\alpha}\left(\right.$ or $\lim _{\leftarrow}\left\{E_{\alpha}, \pi_{\alpha, \beta}\right\}$ or the generalized intersection [5, page 439] $\left.\bigcap_{\alpha \in I} E_{\alpha}\right)$.

Existence in Section 2 is based on the following fixed point results in the literature $[1,6]$.

Theorem 1.2 [6, Theorem 3.9]. Let $U$ be an open subset in a Banach space $(X,\|\cdot\|)$ and $F: \bar{U} \rightarrow X$. Assume $0 \in U$ and suppose there exists a continuous nondecreasing function $\phi:[0, \infty) \rightarrow[0, \infty)$ satisfying $\phi(z)<z$ for $z>0$ such that $\|F x-F y\| \leq \phi(\|x-y\|)$ for all $x, y \in \bar{U}$. In addition, assume $F(\bar{U})$ is bounded and $x \neq \lambda F x$ for $x \in \partial U$ and $\lambda \in(0,1)$. Then $F$ has a fixed point in $\bar{U}$.

Theorem 1.3 [1, Theorem 2.3 (and Remark 2.1)]. Let $U$ be an open subset in a Banach space $(X,\|\cdot\|)$ and $F: \bar{U} \rightarrow \mathbb{C}(X)$ a closed map (i.e., has closed graph); here $\mathbb{C}(X)$ denotes the family of nonempty closed subsets of $X$. Assume $0 \in U$ and suppose there exists a continuous strictly increasing function $\phi:[0, \infty) \rightarrow[0, \infty)$ satisfying $\phi(z)<z$ for $z>0$ such that $H(F x, F y) \leq \phi(\|x-y\|)$ for all $x, y \in \bar{U}$. In addition, assume the following conditions hold:

$$
\begin{aligned}
& \Phi:[0, \infty) \longrightarrow[0, \infty) \text {, given by } \Phi(x)=x-\phi(x) \text {, is strictly increasing, } \\
& \Phi^{-1}(a)+\Phi^{-1}(b) \leq \Phi^{-1}(a+b) \quad \text { for } a, b \geq 0, \\
& \sum_{i=0}^{\infty} \phi^{i}(t)<\infty \quad \text { for } t>0 \\
& \sum_{i=1}^{\infty} \phi^{i}(x-\phi(x)) \leq \phi(x) \quad \text { for } x>0, \\
& F(\bar{U}) \text { is bounded, } \\
& x \notin \lambda F x \quad \text { for } x \in \partial U, \lambda \in(0,1) .
\end{aligned}
$$

Then $F$ has a fixed point in $\bar{U}$. 
Remark 1.4. In fact, the assumption that $F$ is closed can be removed in Theorem 1.3. In $[1$, Theorem 2.3], we assume a more general contractive condition and the map $G: \bar{U} \times$ $[0,1] \rightarrow \mathbb{C}(X)$ (given by $G(x, \lambda)=\lambda F x$ in our situation) was assumed to be closed in order to guarantee that if $\left\{x_{n}\right\}_{1}^{\infty} \subseteq \bar{U},\{\lambda\}_{1}^{\infty} \subseteq[0,1]$ with $x_{n} \in G\left(x_{n}, \lambda_{n}\right)$ and $\left(x_{n}, \lambda_{n}\right) \rightarrow(x, \lambda)$, then $x \in G(x, \lambda)$. However, this is automatically true in Theorem 1.3 since the contractive condition and (1.8) guarantee that $G$ is continuous in the Hausdorff metric and as a result,

$$
\operatorname{dist}(x, G(x, \lambda)) \leq d\left(x, x_{n}\right)+H\left(G\left(x_{n}, \lambda_{n}\right), G(x, \lambda)\right) .
$$

Remark 1.5. If $\phi(t)=k t, 0 \leq k<1$, then trivially (1.2)-(1.7) hold.

\section{Fixed point theory in Fréchet spaces}

Let $E=\left(E,\left\{|\cdot|_{n}\right\}_{n \in \mathbb{N}}\right)$ be a Fréchet space with the topology generated by a family of seminorms $\left\{|\cdot|_{n}: n \in \mathbb{N}\right\}$. We assume that the family of seminorms satisfies

$$
|x|_{1} \leq|x|_{2} \leq|x|_{3} \leq \cdots \quad \text { for every } x \in E \text {. }
$$

A subset $X$ of $E$ is bounded if for every $n \in \mathbb{N}$ there exists $r_{n}>0$ such that $|x|_{n} \leq r_{n}$ for all $x \in X$. To $E$ we associate a sequence of Banach spaces $\left\{\left(\mathbf{E}_{n},|\cdot|_{n}\right)\right\}$ described as follows. For every $n \in \mathbb{N}$, we consider the equivalence relation $\sim_{n}$ defined by

$$
x \sim_{n} y \quad \text { iff }|x-y|_{n}=0 .
$$

We denote by $\mathbf{E}^{n}=\left(E / \sim_{n},|\cdot|_{n}\right)$ the quotient space, and by $\left(\mathbf{E}_{n},|\cdot|_{n}\right)$ the completion of $\mathbf{E}^{n}$ with respect to $|\cdot|_{n}$ (the norm on $\mathbf{E}^{n}$ induced by $|\cdot|_{n}$ and its extension to $\mathbf{E}_{n}$ are still denoted by $\left.|\cdot|_{n}\right)$. This construction defines a continuous map $\mu_{n}: E \rightarrow \mathbf{E}_{n}$. Now since (2.1) is satisfied, the seminorm $|\cdot|_{n}$ induces a seminorm on $\mathbf{E}_{m}$ for every $m \geq n$ (again this seminorm is denoted by $|\cdot|_{n}$ ). Also (2.2) defines an equivalence relation on $\mathbf{E}_{m}$ from which we obtain a continuous map $\mu_{n, m}: \mathbf{E}_{m} \rightarrow \mathbf{E}_{n}$ since $\mathbf{E}_{m} / \sim_{n}$ can be regarded as a subset of $\mathbf{E}_{n}$. We now assume the following condition holds: for each $n \in \mathbb{N}$, there exists a Banach $\operatorname{space}\left(E_{n},|\cdot|_{n}\right)$ and an isomorphism (between normed spaces) $j_{n}: \mathbf{E}_{n} \longrightarrow E_{n}$.

Remark 2.1. (i) For convenience, the norm on $E_{n}$ is denoted by $|\cdot|_{n}$.

(ii) Usually in applications, $\mathbf{E}_{n}=\mathbf{E}^{n}$ for each $n \in \mathbb{N}$.

(iii) Note that if $x \in \mathbf{E}_{n}$ (or $\mathbf{E}^{n}$ ), then $x \in E$. However, if $x \in E_{n}$, then $x$ is not necessarily in $E$ and in fact, $E_{n}$ is easier to use in applications (even though $E_{n}$ is isomorphic to $\mathbf{E}_{n}$ ). For example, if $E=\mathbb{C}[0, \infty)$, then $\mathbf{E}^{n}$ consists of the class of functions in $E$ which coincide on the interval $[0, n]$ and $E_{n}=\mathbb{C}[0, n]$.

Finally, we assume

$$
E_{1} \supseteq E_{2} \supseteq \cdots \text { and for each } n \in \mathbb{N}, \quad|x|_{n} \leq|x|_{n+1} \forall x \in E_{n+1} \text {. }
$$

Let $\lim _{\leftarrow} E_{n}$ (or $\bigcap_{1}^{\infty} E_{n}$ where $\bigcap_{1}^{\infty}$ is the generalized intersection [5]) denote the projective limit of $\left\{E_{n}\right\}_{n \in \mathbb{N}}$ (note that $\pi_{n, m}=j_{n} \mu_{n, m} j_{m}^{-1}: E_{m} \rightarrow E_{n}$ for $m \geq n$ ) and note that $\lim _{\leftarrow} E_{n} \cong$ $E$, so for convenience, we write $E=\lim _{\leftarrow} E_{n}$. 
For each $X \subseteq E$ and each $n \in \mathbb{N}$, we set $X_{n}=j_{n} \mu_{n}(X)$ and we let $\overline{X_{n}}$ and $\partial X_{n}$ denote, respectively, the closure and the boundary of $X_{n}$ with respect to $|\cdot|_{n}$ in $E_{n}$. Also the pseudo-interior of $X$ is defined by [4]

$$
\text { pseudo- } \operatorname{intt}(X)=\left\{x \in X: j_{n} \mu_{n}(x) \in \overline{X_{n}} \backslash \partial X_{n} \text { for every } n \in \mathbb{N}\right\} \text {. }
$$

Also, here $H_{n}$ and diam $_{n}$ denote the Hausdorff metric and the diameter induced by $|\cdot|_{n}$ on $E_{n}$.

We begin with single-valued maps and present two results. The first was motivated by Volterra type operators.

Theorem 2.2. Let $E$ and $E_{n}$ be as described above and let $F: X \rightarrow E$ with $X \subseteq E$ and for each $n \in \mathbb{N}$ assume that $F: \overline{X_{n}} \rightarrow E_{n}$. Suppose the following conditions are satisfied:

(a) $0 \in$ pseudo-intt $(X)$,

(b) for each $n \in \mathbb{N}, F\left(\overline{X_{n}}\right)$ is bounded,

(c) for each $n \in \mathbb{N}, F: \overline{X_{n}} \rightarrow E_{n}$ and there exists a continuous nondecreasing function $\phi_{n}:[0, \infty) \rightarrow[0, \infty)$ satisfying $\phi_{n}(z)<z$ for $z>0$ such that $|F x-F y|_{n} \leq \phi_{n}(\mid x-$ $\left.\left.y\right|_{n}\right)$ for all $x, y \in \overline{X_{n}}$ for each $n \in \mathbb{N}, y \neq \lambda F y$, in $E_{n}$ for all $\lambda \in(0,1), y \in \partial X_{n}$,

(d) for each $n \in\{2,3, \ldots\}$, if $y \in \overline{X_{n}}$ solves $y=F y$ in $E_{n}$, then $y \in \overline{X_{k}}$ for $k \in\{1, \ldots, n-1\}$. Then $F$ has a fixed point in $E$.

Remark 2.3. If $F(X)$ is bounded, then clearly Theorem 2.2(b) holds.

Proof. Fix $n \in \mathbb{N}$. From Theorem 1.2, there exists $y_{n} \in \overline{X_{n}}$ with $y_{n}=F y_{n}$ (note that $0 \in$ $\overline{X_{n}} \backslash \partial X_{n}$ and $F\left(\overline{X_{n}}\right)$ is bounded). Let us look at $\left\{y_{n}\right\}_{n \in \mathbb{N}}$. Notice that $y_{1} \in \overline{X_{1}}$ and $y_{k} \in$ $\overline{X_{1}}$ for $k \in \mathbb{N} \backslash\{1\}$ from Theorem 2.2(d). As a result, $y_{n} \in \overline{X_{1}}$ for $n \in \mathbb{N}, y_{n}=F y_{n}$ in $E_{n}$ together with Theorem 1.1 implies there is a subsequence $\mathbb{N}_{1}^{\star}$ of $\mathbb{N}$ and a $z_{1} \in \overline{X_{1}}$ with $y_{n} \rightarrow z_{1}$ in $E_{1}$ as $n \rightarrow \infty$ in $\mathbb{N}_{1}^{\star}$. Let $\mathbb{N}_{1}=\mathbb{N}_{1}^{\star} \backslash\{1\}$. Now $y_{n} \in \overline{X_{2}}$ for $n \in \mathbb{N}_{1}$ together with Theorem 1.1 guarantees that there exists a subsequence $\mathbb{N}_{2}^{\star}$ of $\mathbb{N}_{1}$ and a $z_{2} \in \overline{X_{2}}$ with $y_{n} \rightarrow z_{2}$ in $E_{2}$ as $n \rightarrow \infty$ in $\mathbb{N}_{2}^{\star}$. Note from (2.3) that $z_{2}=z_{1}$ in $E_{1}$ since $\mathbb{N}_{2}^{\star} \subseteq \mathbb{N}_{1}$. Let $\mathbb{N}_{2}=\mathbb{N}_{2}^{\star} \backslash\{2\}$. Proceed inductively to obtain subsequences of integers

$$
\begin{gathered}
\mathbb{N}_{1}^{\star} \supseteq \mathbb{N}_{2}^{\star} \supseteq \cdots, \\
\mathbb{N}_{k}^{\star} \subseteq\{k, k+1, \ldots\},
\end{gathered}
$$

and $z_{k} \in \overline{X_{k}}$ with $y_{n} \rightarrow z_{k}$ in $E_{k}$ as $n \rightarrow \infty$ in $\mathbb{N}_{k}^{\star}$. Note that $z_{k+1}=z_{k}$ in $E_{k}$ for $k \in\{1,2, \ldots\}$. Also let $\mathbb{N}_{k}=\mathbb{N}_{k}^{\star} \backslash\{k\}$.

Fix $k \in \mathbb{N}$. Let $y=z_{k}$ in $E_{k}$. Notice that $y$ is well defined and $y \in \lim _{\leftarrow} E_{n}=E$. Now $y_{n}=F y_{n}$ in $E_{n}$ for $n \in \mathbb{N}_{k}$ and $y_{n} \rightarrow y$ in $E_{k}$ as $n \rightarrow \infty$ in $\mathbb{N}_{k}$ (since $y=z_{k}$ in $E_{k}$ ) together with the fact that $F: \overline{X_{k}} \rightarrow E_{k}$ is continuous (note that $y_{n} \in \overline{X_{k}}$ for $n \in \mathbb{N}_{k}$ ) implies $y=F y$ in $E_{k}$. We can do this for each $k \in \mathbb{N}$, so $y=F y$ in $E$.

Our next result was motivated by contractions considered in [3]. In this case, the map $F_{n}$ will be related to $F$ by the closure property Theorem $2.4(\mathrm{f})$. 
Theorem 2.4. Let $E$ and $E_{n}$ be as described in the beginning of Section 2 and let $F: X \rightarrow E$ with $X \subseteq E$. Also for each $n \in \mathbb{N}$ assume there exists $F_{n}: \overline{X_{n}} \rightarrow E_{n}$. Suppose the following conditions are satisfied:

(a) $0 \in$ pseudo- $\operatorname{intt}(X)$,

(b) $\overline{X_{1}} \supseteq \overline{X_{2}} \supseteq \cdots$,

(c) for each $n \in \mathbb{N}, F_{n}\left(\overline{X_{n}}\right)$ is bounded, for each $n \in \mathbb{N}, F_{n}: \overline{X_{n}} \rightarrow E_{n}$ and there exists a continuous nondecreasing function $\phi_{n}:[0, \infty) \rightarrow[0, \infty)$ satisfying $\phi_{n}(z)<z$ for $z>$ 0 such that $\left|F_{n} x-F_{n} y\right|_{n} \leq \phi_{n}\left(|x-y|_{n}\right)$ for all $x, y \in \overline{X_{n}}$ for each $n \in \mathbb{N}, y \neq \lambda F_{n} y$ in $E_{n}$ for all $\lambda \in(0,1), y \in \partial X_{n}$,

(d) for each $n \in \mathbb{N}$, the map $\mathcal{K}_{n}: \overline{X_{n}} \rightarrow 2^{E_{n}}$ given by $\mathscr{K}_{n}(y)=\bigcup_{m=n}^{\infty} F_{m}(y)$ (see Remark 2.5) satisfies $H_{n}\left(\mathscr{K}_{n}(x), \mathscr{K}_{n}(y)\right) \leq \psi_{n}\left(|x-y|_{n}\right)$ for all $x, y \in \overline{X_{n}}$; here $\psi_{n}:[0, \infty) \rightarrow$ $[0, \infty)$ is continuous, $\psi_{n}(z)<z$ for $z>0$ with the map $\Psi_{n}:[0, \infty) \rightarrow[0, \infty)$, defined by $\Psi_{n}(x)=x-\psi_{n}(x)$, strictly increasing,

(e) for each $k \in \mathbb{N}$, for every $\epsilon>0$, and sequence $\left\{x_{n}\right\}_{n \in S}, S=\{k, k+1, k+2, \ldots\}$, with $x_{n} \in \overline{X_{n}}$ and $x_{n} \in \mathscr{K}_{n} x_{n}$ in $E_{n}$, there exists $n_{k} \in S$ such that $\operatorname{diam}_{k}\left(\mathscr{K}_{k} x_{n}\right)<\epsilon$ for each $n \in S$ with $n \geq n_{k}$,

(f) if there exists $w \in E$ and a sequence $\left\{y_{n}\right\}_{n \in \mathbb{N}}$ with $y_{n} \in \overline{X_{n}}$ and $y_{n}=F_{n} y_{n}$ in $E_{n}$ such that for every $k \in \mathbb{N}$ with $y_{n} \rightarrow w$ in $E_{k}$ as $n \rightarrow \infty$ in $S=\{k+1, k+2, \ldots\}$, then $w=F w$ in $E$.

Then $F$ has a fixed point in $E$.

Remark 2.5. The definition of $\mathscr{K}_{n}$ in Theorem 2.4(d) is as follows. If $y \in \overline{X_{n}}$ and $y \notin \overline{X_{n+1}}$, then $\mathcal{K}_{n}(y)=F_{n}(y)$, whereas if $y \in \overline{X_{n+1}}$ and $y \notin \overline{X_{n+2}}$, then $\mathcal{K}_{n}(y)=F_{n}(y) \cup F_{n+1}(y)$, and so on.

Proof. Fix $n \in \mathbb{N}$. From Theorem 1.2 there exists $y_{n} \in \overline{X_{n}}$ with $y_{n}=F_{n} y_{n}$ in $E_{n}$. Let us look at $\left\{y_{n}\right\}_{n \in \mathbb{N}}$. From Theorem 2.4(b) we know that $y_{n} \in \overline{X_{1}}$ for $n \in \mathbb{N}$. Note as well that $y_{n} \in \mathscr{K}_{1} y_{n}$ for $n \in \mathbb{N}$ since $|x|_{1} \leq|x|_{n}$ for all $x \in E_{n}$ and $y_{n}=F_{n} y_{n}$ in $E_{n}$. We claim

$$
\exists z_{1} \in E_{1} \quad \text { with } y_{n} \longrightarrow z_{1} \text { in } E_{1}, \quad n \longrightarrow \infty \text { in } \mathbb{N} \text {. }
$$

To see this, let $\epsilon>0$ be given. Let $m, n \in \mathbb{N}$. It is easy to see, since $y_{n} \in \mathscr{K}_{1} y_{n}$ and $y_{m} \in$ $\mathcal{Y}_{1} y_{m}$, that

$$
\left|y_{n}-y_{m}\right|_{1} \leq H_{1}\left(\mathscr{K}_{1} y_{n}, \mathscr{K}_{1} y_{m}\right)+\operatorname{diam}_{1}\left(\mathscr{K}_{1} y_{n}\right)+\operatorname{diam}_{1}\left(\mathscr{K}_{1} y_{m}\right)
$$

so Theorem 2.4(d) yields

$$
\left|y_{n}-y_{m}\right|_{1} \leq \Psi_{1}^{-1}\left(\operatorname{diam}_{1}\left(\mathscr{K}_{1} y_{n}\right)+\operatorname{diam}_{1}\left(\mathscr{K}_{1} y_{m}\right)\right)
$$

Now Theorem 2.4(e) guarantees that there exists $n_{1} \in \mathbb{N}$ such that

$$
\left|y_{n}-y_{m}\right|_{1} \leq \Psi_{1}^{-1}(2 \epsilon) \text { for } m, n \geq n_{1} \text {. }
$$

Consequently, $\left\{y_{n}\right\}_{n \in \mathbb{N}}$ is Cauchy, so (2.6) holds. Let $\mathbb{N}_{1}=\mathbb{N} \backslash\{1\}$. 
Now $y_{n} \in \mathscr{K}_{2} y_{n}$ for $n \in \mathbb{N}_{1}$. Let $m, n \in \mathbb{N}_{1}$ and since $y_{n} \in \mathscr{K}_{2} y_{n}$ and $y_{m} \in \mathscr{K}_{2} y_{m}$ we have

$$
\left|y_{n}-y_{m}\right|_{2} \leq \Psi_{2}^{-1}\left(\operatorname{diam}_{2}\left(\mathscr{K}_{2} y_{n}\right)+\operatorname{diam}_{2}\left(\mathscr{K}_{2} y_{m}\right)\right)
$$

This together with Theorem 2.4(e) guarantees that $\left\{y_{n}\right\}_{n \in \mathbb{N}_{1}}$ is Cauchy, so there exists a $z_{2} \in E_{2}$ with $y_{n} \rightarrow z_{2}$ in $E_{2}$ as $n \rightarrow \infty$ in $\mathbb{N}_{1}$. Note that $z_{2}=z_{1}$ in $E_{1}$ since $\mathbb{N}_{1} \subseteq \mathbb{N}$. Let $\mathbb{N}_{2}=$ $\mathbb{N}_{1} \backslash\{2\}$. Proceed inductively to obtain $z_{k} \in E_{k}$ with $y_{n} \rightarrow z_{k}$ in $E_{k}$ as $n \rightarrow \infty$ in $\mathbb{N}_{k-1}=$ $\{k, k+1, \ldots\}$. Note that $z_{k+1}=z_{k}$ in $E_{k}$ for $k \in \mathbb{N}$. Also let $\mathbb{N}_{k}=\mathbb{N}_{k-1} \backslash\{k\}$.

Fix $k \in \mathbb{N}$. Let $y=z_{k}$ in $E_{k}$. Notice that $y$ is well defined and $y \in \lim _{\leftarrow} E_{n}=E$. Now $y_{n}=F_{n} y_{n}$ in $E_{n}$ for $n \in \mathbb{N}_{k}$ and $y_{n} \rightarrow y$ in $E_{k}$ as $n \rightarrow \infty$ in $\mathbb{N}_{k}$ (since $y=z_{k}$ in $E_{k}$ ) together with Theorem 2.4(f) implies $y=F y$ in $E$.

Our next two results are for multivalued maps.

Theorem 2.6. Let $E$ and $E_{n}$ be as described above and let $F: X \rightarrow 2^{E}$ with $X \subseteq E$ and for each $n \in \mathbb{N}$, assume $F: \overline{X_{n}} \rightarrow \mathbb{C}\left(E_{n}\right)$. Suppose the following conditions are satisfied:

(a) $0 \in$ pseudo- $\operatorname{intt}(X)$,

(b) for each $n \in \mathbb{N}, F\left(\overline{X_{n}}\right)$ is bounded,

(c) for each $n \in \mathbb{N}, F: \overline{X_{n}} \rightarrow \mathbb{C}\left(E_{n}\right)$, and there exists a continuous strictly increasing function $\phi_{n}:[0, \infty) \rightarrow[0, \infty)$ satisfying $\phi_{n}(z)<z$ for $z>0$ such that $H_{n}(F x, F y) \leq$ $\phi_{n}\left(|x-y|_{n}\right)$ for all $x, y \in \overline{X_{n}}$

(d) for each $n \in \mathbb{N}$, the map $\Phi_{n}:[0, \infty) \rightarrow[0, \infty)$ given by $\Phi_{n}(x)=x-\phi_{n}(x)$ is strictly increasing, $\Phi_{n}^{-1}(a)+\Phi_{n}^{-1}(b) \leq \Phi_{n}^{-1}(a+b)$ for $a, b \geq 0$, with $\sum_{i=0}^{\infty} \phi_{n}^{i}(t)<\infty$ for $t>$ 0 and $\sum_{i=1}^{\infty} \phi_{n}^{i}(x-\phi(x)) \leq \phi_{n}(x)$ for $x>0$,

(e) for each $n \in \mathbb{N}, y \notin \lambda F y$ in $E_{n}$ for all $\lambda \in(0,1), y \in \partial X_{n}$,

(f) for each $n \in\{2,3, \ldots\}$, if $y \in \overline{X_{n}}$ solves $y \in F y$ in $E_{n}$, then $y \in \overline{X_{k}}$ for $k \in\{1, \ldots, n-1\}$,

(g) for each $k \in \mathbb{N}$, for every $\epsilon>0$ and sequence $\left\{x_{n}\right\}_{n \in S}, S=\{k, k+1, k+2, \ldots\}$, with $x_{n} \in \overline{X_{n}}$ and $x_{n} \in F x_{n}$ in $E_{n}$ there exists $n_{k} \in S$ such that $\operatorname{diam}_{k}\left(F x_{n}\right)<\epsilon$ for each $n \in S$ with $n \geq n_{k}$.

Then $F$ has a fixed point in $E$.

Proof. Fix $n \in \mathbb{N}$. From Theorem 1.3 (and Remark 1.4) there exists $y_{n} \in \overline{X_{n}}$ with $y_{n} \in F y_{n}$ in $E_{n}$. Let us look at $\left\{y_{n}\right\}_{n \in \mathbb{N}}$. Notice that $y_{n} \in \overline{X_{1}}$ for $n \in \mathbb{N}$ from Theorem 2.6(f). Let $\epsilon>0$ be given and $m, n \in \mathbb{N}$. Now since $y_{n} \in F y_{n}$ and $y_{m} \in F y_{m}$, we have

$$
\left|y_{n}-y_{m}\right|_{1} \leq H_{1}\left(F y_{n}, F y_{m}\right)+\operatorname{diam}_{1}\left(F y_{n}\right)+\operatorname{diam}_{1}\left(F y_{m}\right)
$$

so

$$
\left|y_{n}-y_{m}\right|_{1} \leq \Phi_{1}^{-1}\left(\operatorname{diam}_{1}\left(F y_{n}\right)+\operatorname{diam}_{1}\left(F y_{m}\right)\right)
$$

This, together with Theorem 2.6(g), guarantees that $\left\{y_{n}\right\}_{n \in \mathbb{N}}$ is Cauchy, so there exists a $z_{1} \in E_{1}$ with $y_{n} \rightarrow z_{1}$ in $E_{1}$ as $n \rightarrow \infty$ in $\mathbb{N}$. Let $\mathbb{N}_{1}=\mathbb{N} \backslash\{1\}$. Proceed inductively to obtain $z_{k} \in E_{k}$ with $y_{n} \rightarrow z_{k}$ in $E_{k}$ as $n \rightarrow \infty$ in $\mathbb{N}_{k-1}=\{k, k+1, \ldots\}$. Note that $z_{k+1}=z_{k}$ in $E_{k}$ for $k \in \mathbb{N}$. Also let $\mathbb{N}_{k}=\mathbb{N}_{k-1} \backslash\{k\}$. 
Fix $k \in \mathbb{N}$. Let $y=z_{k}$ in $E_{k}$. Notice that $y_{n} \in F y_{n}$ in $E_{n}$ for $n \in \mathbb{N}_{k}$ and $y_{n} \rightarrow y$ in $E_{k}$ as $n \rightarrow \infty$ in $\mathbb{N}_{k}$ together with Remark 1.4 (note that $F: \overline{X_{k}} \rightarrow \mathbb{C}\left(E_{k}\right)$ ) implies $y \in F y$ in $E_{k}$. We can do this for each $k \in \mathbb{N}$, so $y \in F y$ in $E$.

Theorem 2.7. Let $E$ and $E_{n}$ be as described in the beginning of Section 2 and let $F: X \rightarrow 2^{E}$ with $X \subseteq E$. Also for each $n \in \mathbb{N}$ assume there exists $F_{n}: \overline{X_{n}} \rightarrow \mathbb{C}\left(E_{n}\right)$. Suppose the following conditions are satisfied:

(a) $0 \in$ pseudo- $\operatorname{intt}(X)$,

(b) $\overline{X_{1}} \supseteq \overline{X_{2}} \supseteq \cdots$,

(c) for each $n \in \mathbb{N}, F_{n}\left(\overline{X_{n}}\right)$ is bounded,

(d) for each $n \in \mathbb{N}, F_{n}: \overline{X_{n}} \rightarrow \mathbb{C}\left(E_{n}\right)$ and there exists a continuous strictly increasing function $\phi_{n}:[0, \infty) \rightarrow[0, \infty)$ satisfying $\phi_{n}(z)<z$ for $z>0$ such that $H_{n}\left(F_{n} x, F_{n} y\right) \leq$ $\phi_{n}\left(|x-y|_{n}\right)$ for all $x, y \in \overline{X_{n}}$

(e) for each $n \in \mathbb{N}$, the map $\Phi_{n}:[0, \infty) \rightarrow[0, \infty)$ given by $\Phi_{n}(x)=x-\phi_{n}(x)$ is strictly increasing, $\Phi_{n}^{-1}(a)+\Phi_{n}^{-1}(b) \leq \Phi_{n}^{-1}(a+b)$ for $a, b \geq 0$, with $\sum_{i=0}^{\infty} \phi_{n}^{i}(t)<\infty$ for $t>$ 0 and $\sum_{i=1}^{\infty} \phi_{n}^{i}(x-\phi(x)) \leq \phi_{n}(x)$ for $x>0$,

(f) for each $n \in \mathbb{N}, y \notin \lambda F_{n} y$ in $E_{n}$ for all $\lambda \in(0,1)$ and $y \in \partial X_{n}$,

(g) for each $n \in \mathbb{N}$, the map $\mathscr{K}_{n}: \overline{X_{n}} \rightarrow 2^{E_{n}}$ given by $\mathscr{K}_{n}(y)=\bigcup_{m=n}^{\infty} F_{m}(y)$ satisfies $H_{n}\left(\mathscr{K}_{n}(x), \mathscr{K}_{n}(y)\right) \leq \psi_{n}\left(|x-y|_{n}\right)$ for all $x, y \in \overline{X_{n}}$; here $\psi_{n}:[0, \infty) \rightarrow[0, \infty)$ is continuous, $\psi_{n}(z)<z$ for $z>0$ with the map $\Psi_{n}:[0, \infty) \rightarrow[0, \infty)$ defined by $\Psi_{n}(x)=$ $x-\psi_{n}(x)$ is strictly increasing,

(h) for each $k \in \mathbb{N}$, for every $\epsilon>0$ and sequence $\left\{x_{n}\right\}_{n \in S}, S=\{k, k+1, k+2, \ldots\}$, with $x_{n} \in \overline{X_{n}}$ and $x_{n} \in \mathscr{K}_{n} x_{n}$ in $E_{n}$ there exists $n_{k} \in S$ such that $\operatorname{diam}_{k}\left(\mathscr{K}_{k} x_{n}\right)<\epsilon$ for each $n \in S$ with $n \geq n_{k}$,

(i) if there exists a $w \in E$ and a sequence $\left\{y_{n}\right\}_{n \in \mathbb{N}}$ with $y_{n} \in \overline{X_{n}}$ and $y_{n} \in F_{n} y_{n}$ in $E_{n}$ such that for every $k \in \mathbb{N}$ with $y_{n} \rightarrow$ win $E_{k}$ as $n \rightarrow \infty$ in $S=\{k+1, k+2, \ldots\}$, then $w \in F w$ in $E$.

Then $F$ has a fixed point in $E$.

Proof. The proof is essentially the same as in Theorem 2.4 (except that here we use Theorem 1.3 (and Remark 1.4) instead of Theorem 1.2).

\section{References}

[1] R. P. Agarwal, J. Dshalalow, and D. O'Regan, Fixed point and homotopy results for generalized contractive maps of Reich type, Applicable Analysis 82 (2003), no. 4, 329-350.

[2] R. P. Agarwal and D. O'Regan, Various homotopy results for generalized single valued contractions on Fréchet spaces, Journal of Concrete and Applicable Mathematics 1 (2003), no. 2, 101-111.

[3] M. Frigon, Fixed point results for generalized contractions in gauge spaces and applications, Proceedings of the American Mathematical Society 128 (2000), no. 10, 2957-2965.

[4] _ Fixed point results for compact maps on closed subsets of Fréchet spaces and applications to differential and integral equations, Bulletin of the Belgian Mathematical Society. Simon Stevin 9 (2002), no. 1, 23-37.

[5] L. V. Kantorovich and G. P. Akilov, Functional Analysis in Normed Spaces, Pergamon Press, Oxford, 1964. 


\section{Multivalued nonlinear contractions}

[6] D. O'Regan, Fixed point theorems for nonlinear operators, Journal of Mathematical Analysis and Applications 202 (1996), no. 2, 413-432.

Ravi P. Agarwal: Department of Mathematical Science, Florida Institute of Technology, Melbourne, FL 32901, USA

E-mail address: agarwal@fit.edu

Donal O'Regan: Department of Mathematics, National University of Ireland, Galway, Ireland

E-mail address: donal.oregan@nuigalway.ie 


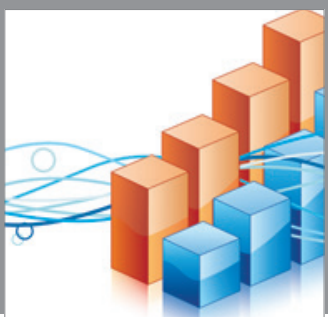

Advances in

Operations Research

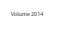

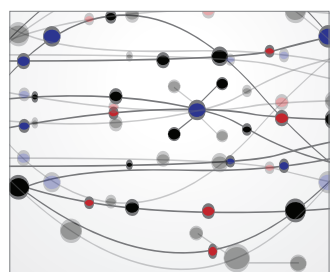

\section{The Scientific} World Journal
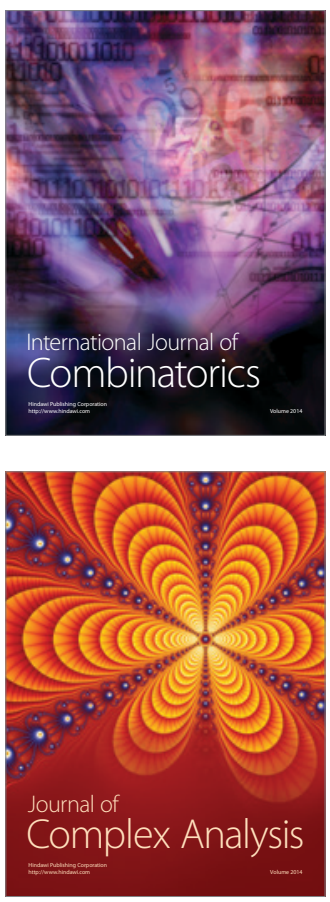

International Journal of

Mathematics and

Mathematical

Sciences
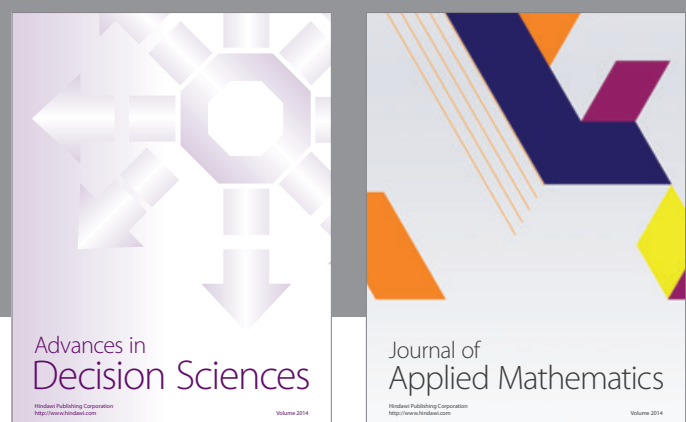

Journal of

Applied Mathematics
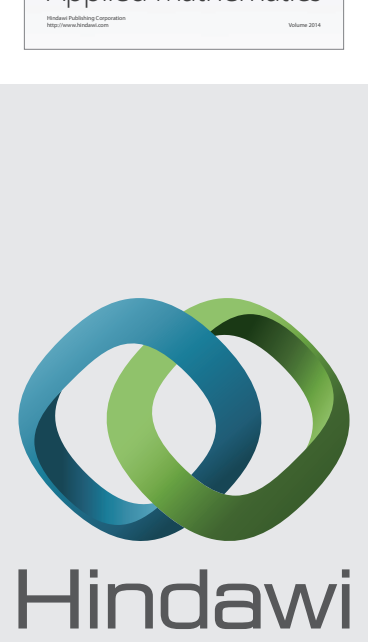

Submit your manuscripts at http://www.hindawi.com
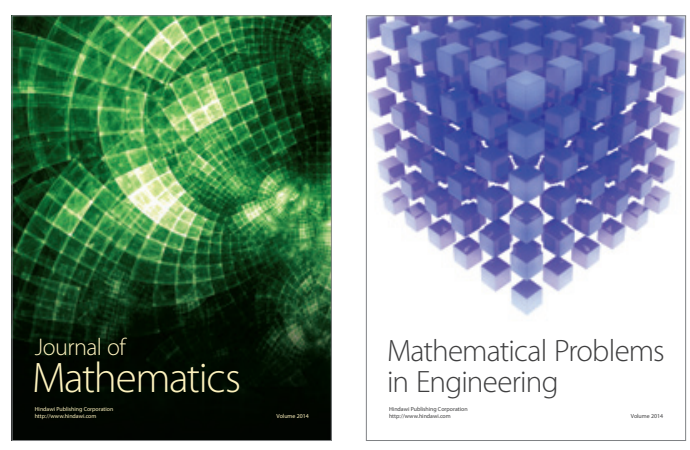

Mathematical Problems in Engineering
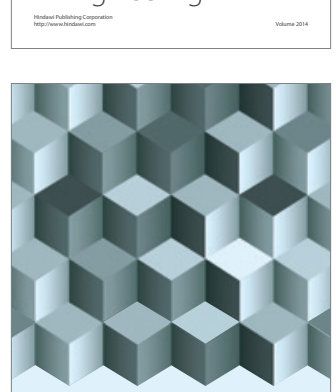

Journal of

Function Spaces
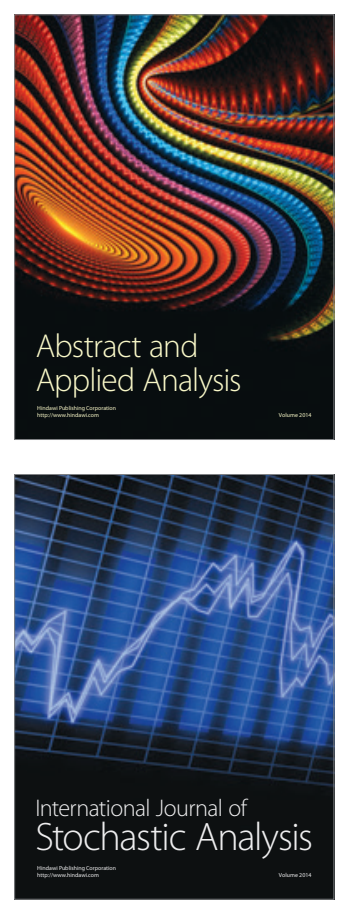

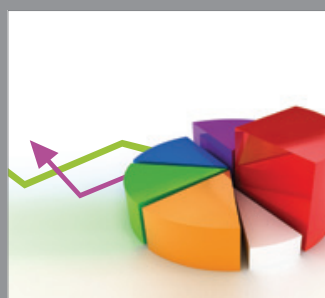

ournal of

Probability and Statistics

Promensencen
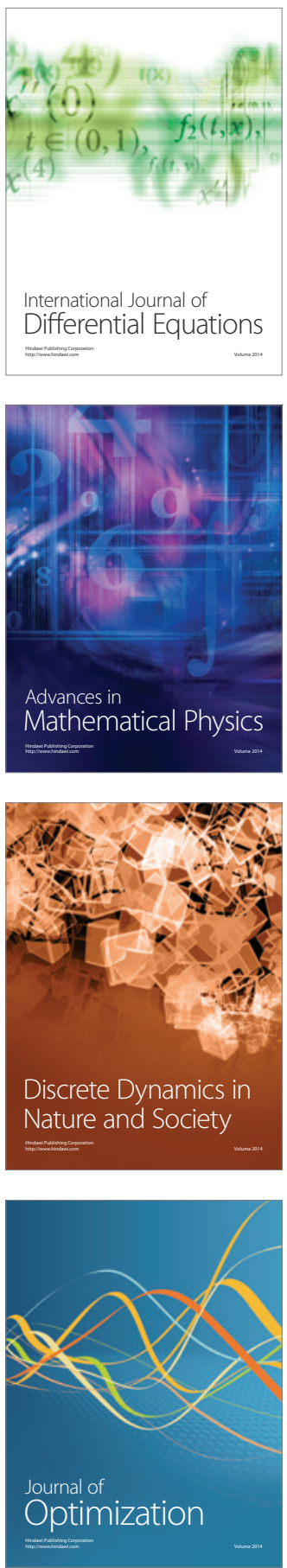